\title{
Letter
}

\section{Imaging Localized Energy States in Silicon-doped InGaN Nanowires Using 4D Electron Microscopy}

Riya Bose, Aniruddha Adhikari, Victor M Burlakov, Guangyu Liu, Md Azimul Haque, Davide Priante, Mohamed N. Hedhili, Nimer Wehbe, Chao Zhao, Haoze Yang, Tien Khee

$\mathrm{Ng}$, Alain Goriely, Osman M. Bakr, Tom Wu, Boon S. Ooi, and Omar F. Mohammed

ACS Energy Lett., Just Accepted Manuscript • DOI: 10.1021/acsenergylett.7b01330 • Publication Date (Web): 23 Jan 2018

Downloaded from http://pubs.acs.org on January 30, 2018

\section{Just Accepted}

"Just Accepted" manuscripts have been peer-reviewed and accepted for publication. They are posted online prior to technical editing, formatting for publication and author proofing. The American Chemical Society provides "Just Accepted" as a free service to the research community to expedite the dissemination of scientific material as soon as possible after acceptance. "Just Accepted" manuscripts appear in full in PDF format accompanied by an HTML abstract. "Just Accepted" manuscripts have been fully peer reviewed, but should not be considered the official version of record. They are accessible to all readers and citable by the Digital Object Identifier (DOI@). "Just Accepted" is an optional service offered to authors. Therefore, the "Just Accepted" Web site may not include all articles that will be published in the journal. After a manuscript is technically edited and formatted, it will be removed from the "Just Accepted" Web site and published as an ASAP article. Note that technical editing may introduce minor changes to the manuscript text and/or graphics which could affect content, and all legal disclaimers and ethical guidelines that apply to the journal pertain. ACS cannot be held responsible for errors or consequences arising from the use of information contained in these "Just Accepted" manuscripts. 


\section{Imaging Localized Energy States in Silicon-Doped}

\section{InGaN Nanowires Using 4D Electron Microscopy}

Riya Bose,,$^{\dagger}$ Aniruddha Adhikari, ${ }^{\dagger}$ Victor M. Burlakov, ${ }^{\ddagger}$ Guangyu Liu, ${ }^{\prime}$ Md Azimul Haque, Davide Priante," Mohamed N. Hedhili," Nimer Wehbe, "Chao Zhao," Haoze Yang, ${ }^{\dagger}$ Tien Khee Ng, ${ }^{\|}$Alain Goriely, ${ }^{\ddagger}$ Osman M. Bakr, ${ }^{\dagger}$ Tom Wu, Boon S. Ooi, ${ }^{\ddagger}$ Omar F. Mohammed ${ }^{\dagger *}$

${ }^{\dagger}$ KAUST Solar Center, Physical Sciences and Engineering Division, King Abdullah University of Science and Technology, Thuwal 23955-6900, Kingdom of Saudi Arabia

${ }^{\ddagger}$ Mathematical Institute, University of Oxford, Woodstock Road, Oxford OX2 6GG, UK

"Photonics Laboratory, Computer, Electrical, and Mathematical Sciences and Engineering, King Abdullah University of Science and Technology, Thuwal, 23955-6900, Kingdom of Saudi Arabia

${ }^{\ddagger}$ Physical Sciences and Engineering Division, King Abdullah University of Science and Technology, Thuwal 23955-6900, Kingdom of Saudi Arabia

"Imaging and Characterization Laboratory, King Abdullah University of Science and Technology, Thuwal 23955-6900, Saudi Arabia

Corresponding author (O.F. M)

*Email: omar.abdelsaboor@kaust.edu.sa 
ABSTRACT. Introducing dopants into InGaN NWs is known to significantly improve their device performances through a variety of mechanisms. However, to further optimize device operation under the influence of large specific surfaces, a thorough knowledge of ultrafast dynamical processes at the surface and interface of these NWs is imperative. Here, we describe the development of four-dimensional scanning ultrafast electron microscopy (4D S-UEM) as an extremely surface-sensitive method to directly visualize in space and time the enormous impact of silicon doping on the surface-carrier dynamics of InGaN NWs. Two time regime dynamics are identified for the first time in a 4D S-UEM experiment: an early time behavior (within 200 picoseconds) associated with the deferred evolution of secondary electrons due to the presence of localized trap states that decrease the electron escape rate and a longer timescale behavior (several ns) marked by accelerated charge carrier recombination. The results are further corroborated by conductivity studies carried out in dark and under illumination.

\section{Table of Contents Graphic}

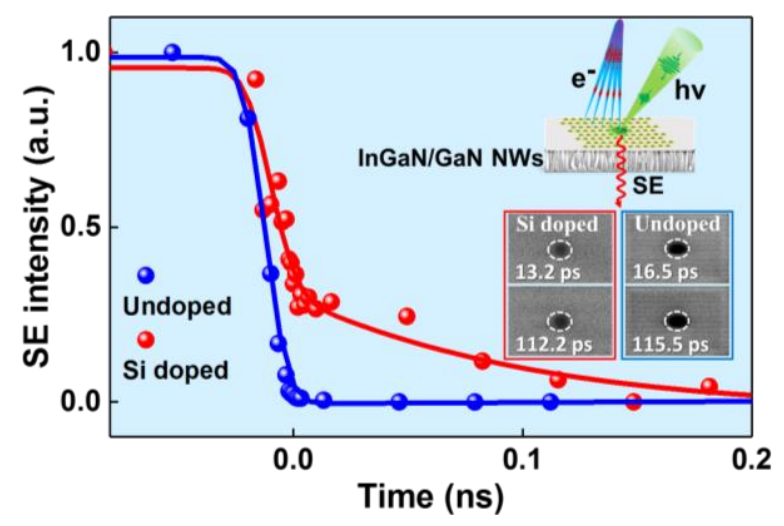


The dynamics of charge carriers at material surfaces and interfaces not only plays a pivotal role in controlling the applicability of nanoscale materials to optoelectronic and microelectronic devices, but is also one of the major challenges in the development of these practical energy technologies. However, real-space and real-time mapping of charge carrier dynamics selectively at material surfaces is beyond the reach of conventional time-resolved spectroscopic or static microscopic methods and has only been enabled by the recent development of four-dimensional scanning ultrafast electron microscopy (4D S-UEM). ${ }^{1-10}$ This technique relies on using photoelectron packets to probe the secondary electrons (SEs) generated from the first few nanometers of the top surface of the material as a result of the interaction of the electron beam with the surface of the photoactive material, which is directly correlated with the excited state charge carrier population and hence the temporal behavior of SEs reflects the charge carrier dynamics, thus opening up a new dimension in imaging techniques: observing the change in charge carrier dynamics explicitly at the surface of the sample in real space and time.

Several approaches have been developed to manipulate charge carrier dynamics in order to enhance the device functionality of photoactive materials; doping is one of the most effective ways among them. ${ }^{11-24}$ In general, insertion of impurity species leads to the formation of additional levels in the bandgap of the host material that not only provide additional relaxation routes for the charge carriers, but may also alter the density of states and the band structure, resulting in significant changes in both the optical and electronic properties of the host material. For example, group III nitride semiconductors, in particular ternary InGaN nanowires (NWs), have gained much attention because of their widespread application as the building block for diverse optoelectronic and photoelectrochemical devices, ${ }^{17-20,25-29}$ stemming from their photostability and widely tunable direct bandgap from UV to near IR regions $(3.4 \mathrm{eV}-0.7 \mathrm{eV})$, 
along with the suitability of the nanowire geometry to allow strain-relaxed growth of complex heterostructures. $^{30-33}$ To further enhance the efficiencies of InGaN-based devices, Si has been introduced as an n-dopant, since it is reported to affect the growth process of NWs, leading to a reduction in structural defects, improvement in their morphology and interface quality, as well as an increase in their thermal stability. ${ }^{34,35}$ Importantly, Si doping can tune the charge carrier dynamics by altering the band structure and carrier recombination. ${ }^{28,36,37}$ Moreover, Si doping leads to screening of the internal piezoelectric field, which in turn reduces the quantum-confined Stark effect, resulting in the decrease of carrier localization, increased radiative recombination rate, photoluminescence intensity, and lowers the threshold power density for obtaining stimulated emission. $^{36,38-40}$

Although efforts have been made to employ 4D S-UEM to monitor the surface charge carrier dynamics of a few technologically important materials, the effect of doping, which is a primary factor in designing the conductivity and optoelectronic properties of the host material, on the charge carrier dynamics remains unexplored. Herein, we investigate the effect of Si doping on the surface charge carrier (as reflected by SE) dynamics of InGaN/GaN NWs using 4D S-UEM. Faster SE kinetics are observed for the Si-doped NWs; however, the growth of the SE signal in the first hundred picosecond regime becomes slower as the Si dopant concentration increases. To understand such an anomaly in the SE kinetics in two different time regions, we conducted numerical simulation and determined that the delay in the contrast evolution of the doped samples can be ascribed to the presence of localized electronic trap states originated from doping, which decreases the electron escape rate, whereas the decay in dark contrast arises from energy losses of the secondary electrons due to their interaction with free and localized carriers generated by dopants. This behavior is also manifested in the photoconductivity of the doped and 
undoped NWs, where the doped NWs show a decrease in the conductivity upon illumination because of the increased charge carrier scattering arising from their increased concentration associated with doping. The current study provides new experimental and theoretical insights into the impact of doping on the charge carrier dynamics and electronic properties of InGaN NWs, which will pave the way to further optimize their properties.

The InGaN/GaN NWs are grown on Si substrates by a plasma-assisted molecular beam epitaxy method. ${ }^{8,41}$ It has already been reported that the use of GaN NWs as templates instead of direct growth of InGaN on Si substrate leads to improved structural properties. ${ }^{28}$ For doping the NWs with $\mathrm{Si}$, two different temperatures are used in order to vary the concentration of the dopant ions, with the higher temperature resulting in a higher dopant concentration. Details of the NW growth procedure are described in the Supporting information.

Figure 1a shows a high-angle annular dark-field scanning transmission electron microscopy (HAADF-STEM) image of the typical InGaN/GaN NWs confirming the formation of the heterostructure, Figure 1(b-d) show the corresponding cross-sectional scanning electron microscopy (SEM) images of both undoped and Si doped NWs. Top-view SEM images of the NWs are provided in the Supporting information (Figure S1). The length and the diameter of the NWs vary in the ranges of $\sim 160-190 \mathrm{~nm}$ and $\sim 20-30 \mathrm{~nm}$, respectively. The NW morphology generally does not change with doping. 


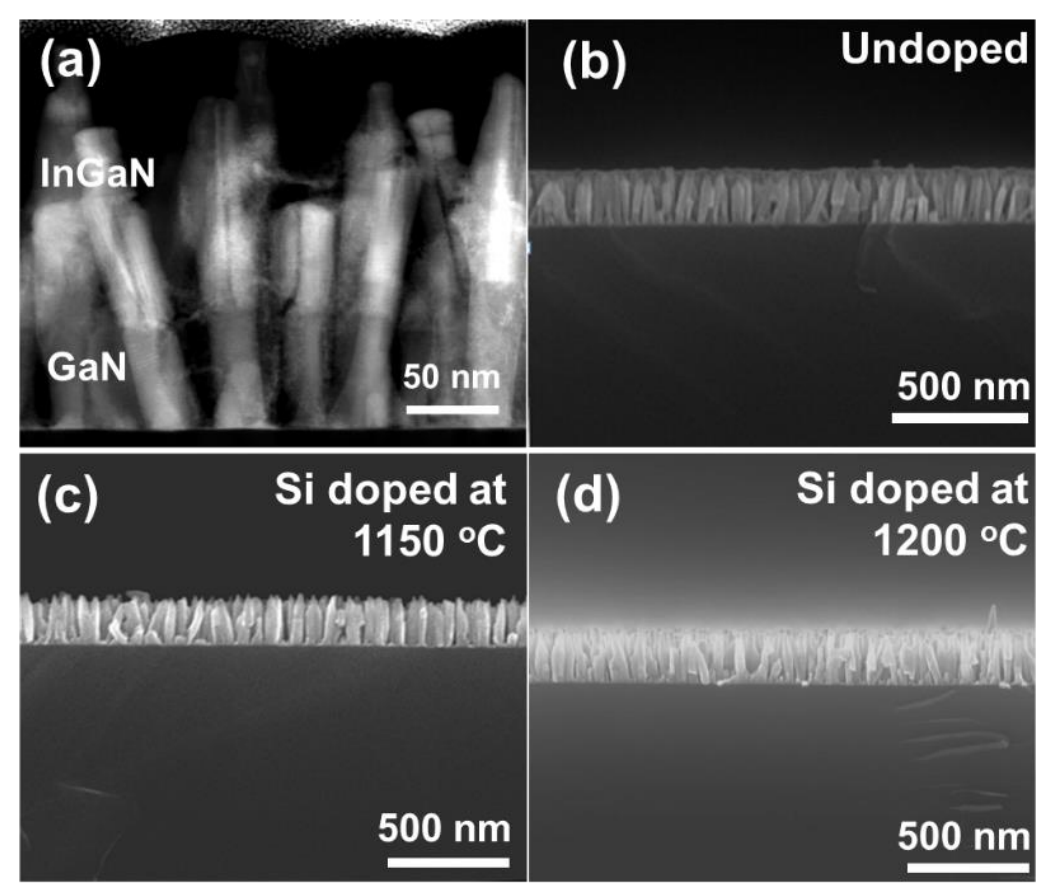

Figure 1. (a) HAADF-STEM image of the InGaN/GaN heterostructures, the InGaN part appear brighter because of the Z-contrast confirming the formation of heterostructures and (b-d) Crosssectional SEM images of the undoped NWs, the NWs doped with Si at $1150{ }^{\circ} \mathrm{C}$ and NWs doped with $\mathrm{Si}$ at $1200{ }^{\circ} \mathrm{C}$, respectively.

Figure 2 shows the time-resolved 4D S-UEM difference images of the undoped and doped NW arrays. The S-UEM technique relies on detecting the changes in the secondary electrons emitted from the top few nanometers of the material surfaces following optical excitation, and probing with a pulsed electron beam, thus precisely revealing the dynamics of the charge carriers on the surface of the material. A schematic diagram of the 4D S-UEM setup and the principle of its operation have been published elsewhere. ${ }^{1,6}$ Briefly, S-UEM combines the output of a fs ClarkMXR fiber laser system with a modified Quanta FEI-650 SEM. The fundamental output of the laser centered at $1030 \mathrm{~nm}$ (pulse, $\sim 270$ fs pulse duration and $200 \mathrm{kHz}$ to $25.4 \mathrm{MHz}$ repetition rate) is split by a 1:1 beam splitter to simultaneously pump a second harmonic generator (HG, Clark-MXR) to produce $515 \mathrm{~nm}$ pulse and a third harmonic generator (HG, Clark-MXR) for 343 
$\mathrm{nm}$ pulse. The $343 \mathrm{~nm}$ output is focused onto the Schottky field emitter tip inside the SEM to generate the pulsed electrons, which are then accelerated towards the sample using a voltage of $30 \mathrm{kV}$. The $515 \mathrm{~nm}$ output enters the microscope through a viewport at a 50-degree angle relative to the surface normal and is focused onto the sample surface at a working distance of 10 $\mathrm{mm}$ from the pole piece of the SEM column to deliver the excitation clocking pulse to the sample. The scanning process of the electron beam proceeds across the surface of the sample, both the laser excited and unexcited regions, in a raster pattern, and the secondary electrons (SEs) emitted from the sample are detected by a positively biased Everhart-Thornley detector. The SE images are obtained at different time delays between the electron and optical pulses as an integration of 64 frames with a dwell time of $300 \mathrm{~ns}$ at each pixel to improve the signal-to-noise ratio. It should be noted that the pump energy of $2.4 \mathrm{eV}$ (corresponding to $515 \mathrm{~nm}$ ) is insufficient to knock out secondary electrons from the sample surface. Finally, all experiments are conducted at a repetition rate of $8 \mathrm{MHz}$ to ensure full recovery of the specimen before the arrival of the next pulse. Contrast-enhanced differences in the SE images from the laser (pump pulse)-irradiated and non-irradiated regions are then extracted with reference to a negative time frame before the arrival of the excitation photon pulse. The evolution of the dark contrast with time for all the samples in the laser illuminated region suggests that while traveling towards the surface, the emitted SEs lose their energy through scattering events with photo-generated carriers; hence, fewer of them are detected by the detector compared to an unilluminated specimen. A similar observation has already been reported for InGaN NWs and GaAs. ${ }^{3,8,42}$ Dense packing of the NWs enables the carrier diffusion across the boundaries of the NWs, and hence, the SE signal spreads beyond the laser-illuminated area. ${ }^{3,8}$ Plotting the SE intensity changes from the center of the laser excitation footprint as a function of the time delays reveals that $\mathrm{Si}$ doping results in 
faster fading away of the dark SE signal, indicating faster carrier relaxation in the doped samples (Figure 3a). One plausible reason is an increase in the number of scattering events in the Si-

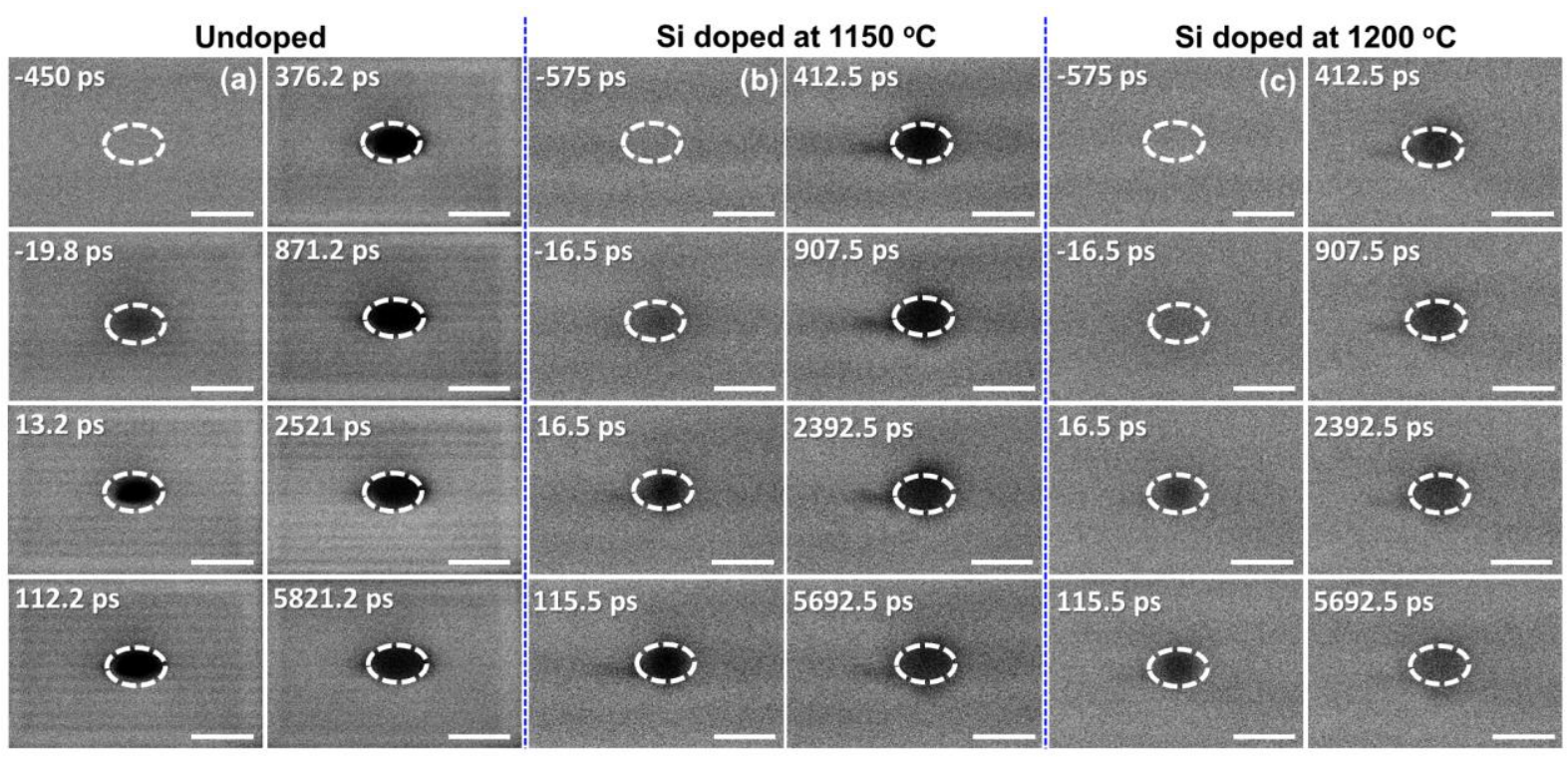

Figure 2. Time-resolved SE difference images at indicated time delays for (a) undoped and (b-c) Si-doped NWs for pump excitation wavelength at $515 \mathrm{~nm}$. The dashed ellipse indicates the laser footprint on the sample. No change in the image contrast is observed at the far negative time delays, indicating the recovery of the system to the initial state after each pump-probe event. The scale bar is $50 \mu \mathrm{m}$.

doped samples because of the increase in the carrier concentration with doping. Reduction in the emission decay lifetime of InGaN emitters with Si doping has been reported in the literature and attributed to screening of the quantum-confined Stark effect and to increasing the electron-hole wave function overlap after doping. ${ }^{43,44}$ Surprisingly, zooming into the SE kinetics at a very early timescale ( 200 ps) reveals slower growth of the dark signal in the doped samples than in the undoped one (Figure 3b), i.e., slower deepening of the dark contrast with time, which is reflective of lesser SE generation as a result of reduced primary electron scattering with fewer 
charge carriers. This effect becomes even more prominent as the concentration of the Si dopant increases.

It is reasonable to explain both the faster decay of the dark contrast on the long time scale and its slower rise on the short time scale within the same numerical framework involving localized energy levels associated with dopants.
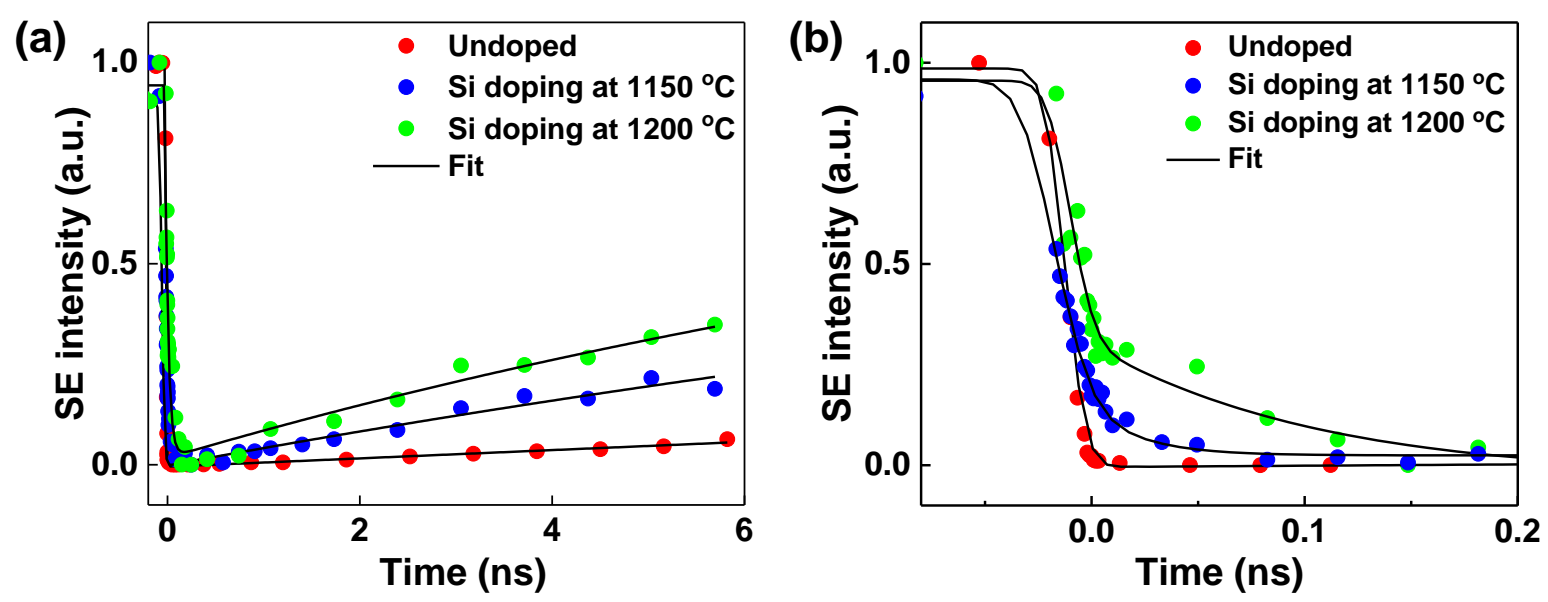

Figure 3. Dynamics of the temporal evolution of the SE intensity at the center of the laser footprint region (a) on $6 \mathrm{~ns}$ time scale and (b) zoomed-in view in the first 200 ps regime. The average time constant for the change of the SE intensity evolution are found to be $1.1 \mathrm{ps}$ for the undoped NWs, 18.8 ps and 34.5 ps for the doped NWs with Si doping temperature $1150{ }^{\circ} \mathrm{C}$ and $1200{ }^{\circ} \mathrm{C}$, respectively. The detailed fitting parameters are provided in the supporting information.

These localized dopant levels can trap electrons, and their occupancy at equilibrium depends on the trapping energy, the total concentrations of free and trapped electrons and the sample temperature. Interaction of the SEs generated by the probe electron pulse with the free and trapped electrons in Si:InGaN can be significantly different. For example, this interaction with the trapped electrons can be more substantial, in which case the trapping dynamics of the photogenerated electrons would be observed as a deferred growth of the dark contrast. 
The trap states can also promote electron-hole recombination, which normally occurs on a longer time scale than the dark contrast growth. This effect would then be observed as a quicker recovery of the dark contrast. The occupancy of the trap states in this case can be in quasiequilibrium with the free electrons. Below, we present a numerical simulation for describing the slower growth and quicker decay of the dark contrast in Si-doped InGaN NWs in reference to the undoped InGaN. In summary, the trap states introduced by Si doping appear to trigger different mechanisms dictating in different time windows: deferred SE emission within 200 ps but accelerated carrier recombination at several ns.

Evolution of the photogenerated carriers can be described by kinetic equations for the concentration of localized (trapped) $n_{L}$ electrons and the concentration $n_{g}$ of photogenerated electrons (and holes)

$$
\begin{aligned}
& \frac{d n_{L}}{d t}=\sigma_{\text {trap }} \cdot\left(n_{g}+n_{b}-n_{L}\right) \cdot\left(N_{L}-n_{L}\right)-n_{L} \cdot r_{e s c}-\gamma_{L} n_{L} n_{g} \\
& \frac{d n_{g}}{d t}=-\gamma_{L} n_{L} n_{g}-\gamma_{f}\left(n_{g}+n_{b}-n_{L}\right) \cdot n_{g}
\end{aligned}
$$

where $n_{b}$ is the background concentration of electrons (n-doping); $N_{L}$ is the concentration of dopants (localized states); $\sigma_{\text {trap }}$ and $r_{\text {esc }}$ are the trapping and detrapping (escape) rates, respectively; and $\gamma_{L}$ and $\gamma_{f}$ are the recombination rates for the trapped and free electrons, respectively. According to the experimental data on dark contrast time evolution, the characteristic time scales for the dark contrast growth and subsequent decay are very different. In particular, dark contrast growth occurs within a fraction of a nanosecond, while the decay is significantly longer, definitely more than 10 ns. This suggests that the growth and the decay kinetics of the dark contrast can be analyzed independently. 


$$
\begin{aligned}
& I_{D C}(t)=P_{1} \cdot\left[P_{2} \cdot N_{L} \cdot\left(1-\exp \left(-r_{e s c} \cdot t\right)\right)+1\right]+1 \text { rise } \\
& I_{D C}(t)=P_{1} \cdot\left[P_{2} \cdot N_{L}+1\right] \cdot \exp \left(-\Gamma \cdot N_{L} \cdot t\right)+1 \quad \text { decay } \\
& P_{1}=\frac{n_{g}(0) \sigma_{f}}{P_{2} \cdot N_{L}+1}, P_{2}=\left(\sigma_{L}-\sigma_{f}\right) \cdot \frac{\sigma_{\text {trap }}}{\sigma_{f} \cdot r_{\text {esc }}}, \Gamma=\gamma_{L} \frac{\sigma_{\text {trap }}}{r_{\text {esc }}} N_{L}+\gamma_{f}
\end{aligned}
$$

Summarizing both the growth and decay of the dark contrast as detailed in the supporting information and transforming the equations to mimic the dependences shown in Figure 3 provide (see Supporting Information). As we can see from these expressions, the decay rate is proportional to $N_{L}$, suggesting a faster decay at higher dopant concentrations, which is exactly what is observed experimentally (see Figure 3a). The dark contrast growth and decay simulated using Eqs (1) are shown in Figure 4(a,b) for $n_{g}(0) \sigma_{f}=-1, P_{2}=5 \cdot 10^{-21} \mathrm{~cm}^{3}, r_{e s c}=20 \mathrm{~ns}^{-1}$, $\Gamma=5^{*} 10^{-22} \mathrm{~ns}^{-1} \mathrm{~cm}^{3}$ and various $N_{L}$ values.

By comparing Figures 3 and 4, we find a good qualitative agreement between the theory and experimental data. This indicates that our assumption about the role of the localized energy levels in changing the time evolution of the dark contrast is appropriate. Accordingly, we can estimate the content of Si doping in InGaN NWs at the higher temperature: most likely, it results in an increase in the dopant concentration $\left(\mathrm{N}_{\mathrm{L}}\right)$ by approximately a factor of 2 .

We further carried out electrical characterization to understand the effect of Si doping on the photoresponse of the InGaN/GaN NWs. As shown in Figure 4c, the dark current of the Si-doped samples is higher than that of the undoped one, which is consistent with the fact that the doped samples have higher carrier concentration. Under illumination, the undoped sample (Figure 4d) exhibited a typical photoresponse (a decrease in resistance under illumination). Interestingly, the photoresponse of the doped samples is to some extent opposite to the undoped counterpart: the resistance slightly increases under illumination, and the effect increases with increasing $\mathrm{Si}$ dopant concentration (Figure 4(e-f)). This can be attributed to carrier-carrier scattering due to the 
high carrier concentration in the case of the Si-doped samples, which is consistent with the secondary electron dynamics observed from 4D S-UEM. Similar observations (a decrease in conductivity under illumination) have already been reported for other semiconductor devices. ${ }^{45-49}$
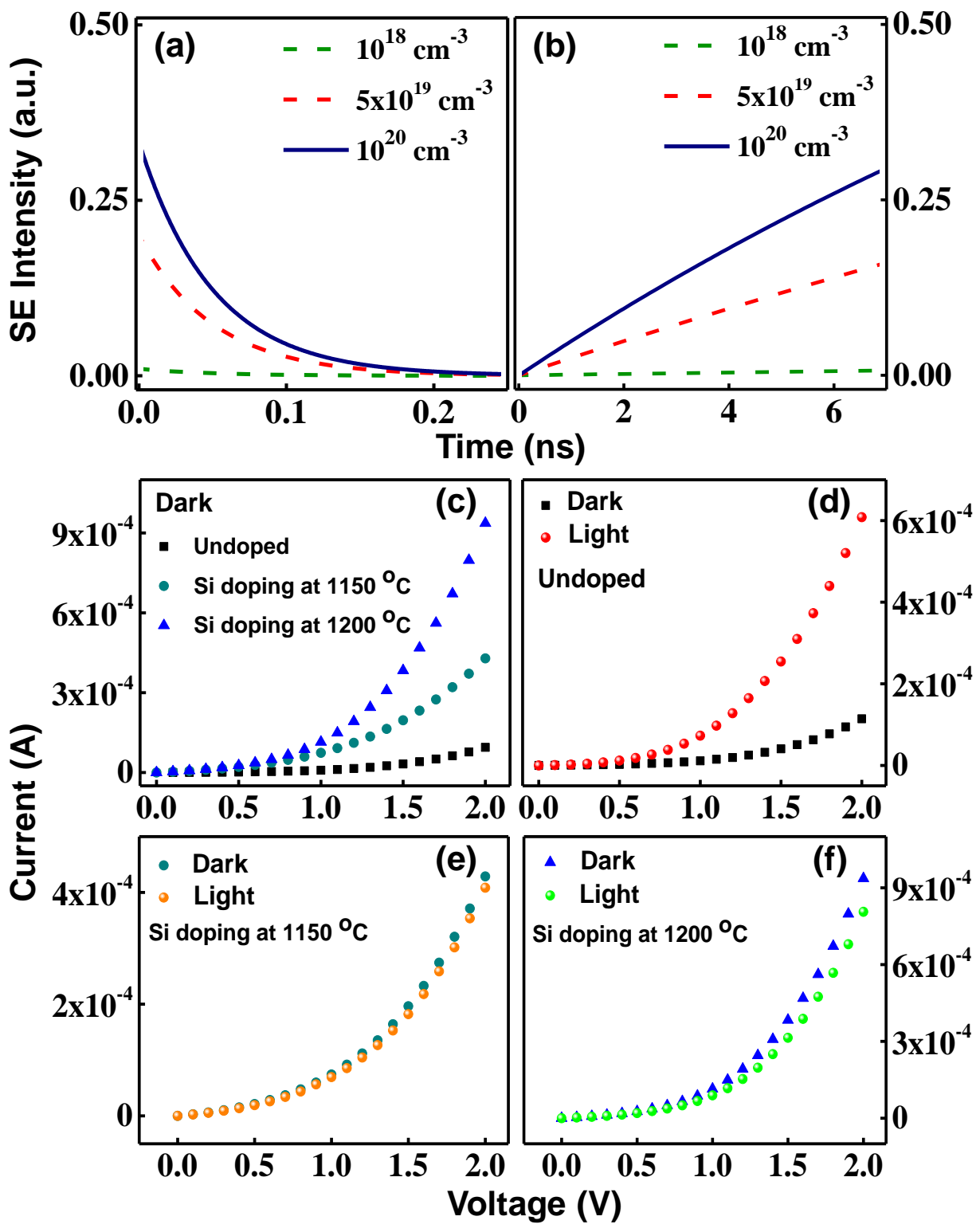

Figure 4. Time dependences of the secondary electron intensity (dark contrast) after the photoexcitation of the samples: (a) growth and (b) decay. The values of $N_{L}$ are $10^{18} \mathrm{~cm}^{-3}$ (green dashed line), $5 \times 10^{19} \mathrm{~cm}^{-3}$ (red dashed line), and $10^{20} \mathrm{~cm}^{-3}$ (solid line). (c) Comparison of the dark current for the undoped and Si-doped InGaN/GaN NW devices. (d-f) Comparison of the dark and photocurrent of the (d) Undoped (e-f) Si doped InGaN/GaN NW devices. 
With the help of numerical simulations (see Supporting Information) as well, focusing on only the sign of the conductivity change under illumination, it can be observed that the effect of illumination has two competing contributions on the photocurrent of the NWs: the one due to the increase in charge carrier concentration is positive, whereas, the other one due to scattering of all electrons on photo-generated holes is negative. The latter contribution dominates for heavily doped samples, as in the case of the specimens we have studied here.

To conclude, we observed the effect of Si doping on the surface charge carrier dynamics of Sidoped InGaN NWs by 4D S-UEM. An interesting behavior of SE contrast evolution was observed; there is a delay in the dark contrast evolution in the fast time regime for the doped samples; however, on a longer time scale, the doped samples show faster decay of the SE signal. To understand such a behavior, we carried out a numerical simulation, which reveals the presence of localized trap states with Si doping that lead to a slower electron release rate and hence a slower growth of the SE signal, whereas on a longer time scale, the scattering of the secondary electron beam with an increased number of charge carriers in the doped samples leads to a faster decay of SE signal. This behavior is also manifested in the photoconductivity of the NWs, whereas the doped NWs show an increase in resistance with illumination because of carrier scattering. Overall, this study provides an in-depth understanding of the impact of Si doping on the charge carrier dynamics of InGaN NWs and will help to further optimize their properties for optoelectronic applications.

\section{ASSOCIATED CONTENT}

Supporting Information. Experimental methods, Instrumentation, device fabrication procedure, detailed numerical simulation and supporting figures. 


\section{AUTHOR INFORMATION}

\section{Corresponding Author}

*Email. omar.abdelsaboor@kaust.edu.sa

\section{ACKNOWLEDGMENT}

The work reported here was supported by King Abdullah University of Science and Technology (KAUST).

\section{REFERENCES}

(1) Mohammed, O. F.; Yang, D.-S.; Pal, S. K.; Zewail, A. H. 4D Scanning Ultrafast Electron Microscopy: Visualization of Materials Surface Dynamics J. Am. Chem. Soc. 2011, $133,7708-7711$.

(2) Bose, R.; Ahmed, G. H.; Alarousu, E.; Parida, M. R.; Abdelhady, A. L.; Bakr, O. M.; Mohammed, O. F. Direct Femtosecond Observation of Charge Carrier Recombination in Ternary Semiconductor Nanocrystals: The Effect of Composition and Shelling J. Phys. Chem. C 2015, 119, 3439-3446.

(3) Khan, J. I.; Adhikari, A.; Sun, J.; Priante, D.; Bose, R.; Shaheen, B. S.; Ng, T. K.; Zhao, C.; Bakr, O. M.; Ooi, B. S. et al. Enhanced Optoelectronic Performance of a Passivated Nanowire-Based Device: Key Information from Real-Space Imaging Using 4D Electron Microscopy Small 2016, 12, 2313-2320.

(4) Adhikari, A.; Eliason, J. K.; Sun, J.; Bose, R.; Flannigan, D. J.; Mohammed, O. F. Four-Dimensional Ultrafast Electron Microscopy: Insights into an Emerging Technique ACS Appl. Mater. Interfaces 2017, 9, 3-16.

(5) Sun, J.; Adhikari, A.; Shaheen, B. S.; Yang, H.; Mohammed, O. F. Mapping Carrier Dynamics on Material Surfaces in Space and Time using Scanning Ultrafast Electron Microscopy J. Phys. Chem. Lett. 2016, 7, 985-994. 
(6) Sun, J.; Melnikov, V. A.; Khan, J. I.; Mohammed, O. F. Real-Space Imaging of Carrier Dynamics of Materials Surfaces by Second-Generation Four-Dimensional Scanning Ultrafast Electron Microscopy J. Phys. Chem. Lett. 2015, 6, 3884-3890.

(7) Bose, R.; Bera, A.; Parida, M. R.; Adhikari, A.; Shaheen, B. S.; Alarousu, E.; Sun, J.; Wu, T.; Bakr, O. M.; Mohammed, O. F. Real-Space Mapping of Surface Trap States in CIGSe Nanocrystals Using 4D Electron Microscopy Nano Lett. 2016, 16, 4417-4423.

(8) Bose, R.; Sun, J.; Khan, J. I.; Shaheen, B. S.; Adhikari, A.; Ng, T. K.; Burlakov, V. M.; Parida, M. R.; Priante, D.; Goriely, A. et al. Real-Space Visualization of Energy Loss and Carrier Diffusion in a Semiconductor Nanowire Array Using 4D Electron Microscopy $A d v$. Mater. 2016, 28, 5106-5111.

(9) Yang, D.-S.; Mohammed, O. F.; Zewail, A. H. Scanning Ultrafast Electron Microscopy. Proc. Natl. Acad. Sci. 2010, 107, 14993-14998.

(10) Shaheen, B. S.; Sun, J.; Yang, D.-S.; Mohammed, O. F. Spatiotemporal Observation of Electron-Impact Dynamics in Photovoltaic Materials Using 4D Electron Microscopy J. Phys. Chem. Lett. 2017, 8, 2455-2462.

(11) Erwin, S. C.; Zu, L.; Haftel, M. I.; Efros, A. L.; Kennedy, T. A.; Norris, D. J. Doping Semiconductor Nanocrystals Nature 2005, 436, 91-94.

(12) Norris, D. J.; Efros, A. L.; Erwin, S. C. Doped Nanocrystals Science 2008, 319, 1776-1779.

(13) Mocatta, D.; Cohen, G.; Schattner, J.; Millo, O.; Rabani, E.; Banin, U. Heavily Doped Semiconductor Nanocrystal Quantum Dots Science 2011, 332, 77-81.

(14) Arkhipov, V. I.; Heremans, P.; Emelianova, E. V.; Bässler, H. Effect of Doping on the Density-of-States Distribution and Carrier Hopping in Disordered Organic Semiconductors Phys. Rev. B 2005, 71, 045214.

(15) Berggren, K. F.; Sernelius, B. E. Band-gap Narrowing in Heavily Doped ManyValley Semiconductors Phys. Rev. B 1981, 24, 1971-1986.

(16) Abram, R. A.; Rees, G. J.; Wilson, B. L. H. Heavily Doped Semiconductors and Devices Adv. Phys. 1978, 27, 799-892.

(17) Koester, R.; Hwang, J.-S.; Salomon, D.; Chen, X.; Bougerol, C.; Barnes, J.-P.; Dang, D. L. S.; Rigutti, L.; de Luna Bugallo, A.; Jacopin, G.; et al. M-Plane Core-Shell 
InGaN/GaN Multiple-Quantum-Wells on GaN Wires for Electroluminescent Devices Nano Lett. 2011, 11, 4839-4845.

(18) Shuji, N.; Masayuki, S.; Shin-ichi, N.; Naruhito, I.; Takao, Y.; Toshio, M.; Hiroyuki, K.; Yasunobu, S. InGaN-Based Multi-Quantum-Well-Structure Laser Diodes Jpn. J. Appl. Phys. 1996, 35, L74-L76.

(19) Chuang, R. W.; Chang, S. P.; Chang, S. J.; Chiou, Y. Z.; Lu, C. Y.; Lin, T. K.; Lin, Y. C.; Kuo, C. F.; Chang, H. M. Gallium Nitride Metal-semiconductor-Metal Photodetectors Prepared on Silicon Substrates J. Appl. Phys. 2007, 102, 073110.

(20) Dong, Y.; Tian, B.; Kempa, T. J.; Lieber, C. M. Coaxial Group III-Nitride Nanowire Photovoltaics Nano Lett. 2009, 9, 2183-2187.

(21) Begum, R.; Parida, M. R.; Abdelhady, A. L.; Murali, B.; Alyami, N. M.; Ahmed, G. H.; Hedhili, M. N.; Bakr, O. M.; Mohammed, O. F. Engineering Interfacial Charge Transfer in $\mathrm{CsPbBr}_{3}$ Perovskite Nanocrystals by Heterovalent Doping J. Am. Chem. Soc. 2017, 139, 731737.

(22) Stavrinadis, A.; Pradhan, S.; Papagiorgis, P.; Itskos, G.; Konstantatos, G. Suppressing Deep Traps in PbS Colloidal Quantum Dots via Facile Iodide Substitutional Doping for Solar Cells with Efficiency >10\% ACS Energy Lett. 2017, 2, 739-744.

(23) Zhang, J.; Shang, M.-h.; Wang, P.; Huang, X.; Xu, J.; Hu, Z.; Zhu, Y.; Han, L. nType Doping and Energy States Tuning in $\mathrm{CH}_{3} \mathrm{NH}_{3} \mathrm{~Pb}_{1-\mathrm{x}} \mathrm{Sb}_{2 \mathrm{x} / 3} \mathrm{I}_{3}$ Perovskite Solar Cells ACS Energy Lett. 2016, 1, 535-541.

(24) Zohar, A.; Levine, I.; Gupta, S.; Davidson, O.; Azulay, D.; Millo, O.; Balberg, I.; Hodes, G.; Cahen, D. What Is the Mechanism of $\mathrm{MAPbI}_{3}$ p-Doping by $\mathrm{I}_{2}$ ? Insights from Optoelectronic Properties ACS Energy Lett. 2017, 2, 2408-2414.

(25) Kim, H.-M.; Cho, Y.-H.; Lee, H.; Kim, S. I.; Ryu, S. R.; Kim, D. Y.; Kang, T. W.; Chung, K. S. High-Brightness Light Emitting Diodes Using Dislocation-Free Indium Gallium Nitride/Gallium Nitride Multiquantum-Well Nanorod Arrays Nano Lett. 2004, 4, 10591062.

(26) Tchernycheva, M.; Messanvi, A.; de Luna Bugallo, A.; Jacopin, G.; Lavenus, P.; Rigutti, L.; Zhang, H.; Halioua, Y.; Julien, F. H.; Eymery, J. et al. Integrated Photonic Platform Based on InGaN/GaN Nanowire Emitters and Detectors Nano Lett. 2014, 14, 3515-3520. 
(27) Hwang, Y. J.; Wu, C. H.; Hahn, C.; Jeong, H. E.; Yang, P. Si/InGaN Core/Shell Hierarchical Nanowire Arrays and their Photoelectrochemical Properties Nano Lett. 2012, 12, 1678-1682.

(28) AlOtaibi, B.; Nguyen, H. P. T.; Zhao, S.; Kibria, M. G.; Fan, S.; Mi, Z. Highly Stable Photoelectrochemical Water Splitting and Hydrogen Generation Using a Double-Band InGaN/GaN Core/Shell Nanowire Photoanode Nano Lett. 2013, 13, 4356-4361.

(29) AlOtaibi, B.; Kong, X.; Vanka, S.; Woo, S. Y.; Pofelski, A.; Oudjedi, F.; Fan, S.; Kibria, M. G.; Botton, G. A.; Ji, W. et al. Photochemical Carbon Dioxide Reduction on MgDoped $\mathrm{Ga}(\mathrm{In}) \mathrm{N}$ Nanowire Arrays under Visible Light Irradiation ACS Energy Lett. 2016, 1, 246252.

(30) Kuykendall, T.; Ulrich, P.; Aloni, S.; Yang, P. Complete Composition Tunability of InGaN Nanowires Using a Combinatorial Approach Nat. Mater. 2007, 6, 951-956.

(31) Yam, F. K.; Hassan, Z. InGaN: An Overview of the Growth Kinetics, Physical Properties and Emission Mechanisms Superlattices Microstruct. 2008, 43, 1-23.

(32) Takashi, M.; Motokazu, Y.; Shuji, N. Characteristics of InGaN-Based UV/Blue/Green/Amber/Red Light-Emitting Diodes Jpn. J. Appl. Phys. 1999, 38, 3976-3981.

(33) Yan, R.; Gargas, D.; Yang, P. Nanowire Photonics Nat. Photonics 2009, 3, 569576.

(34) Kamimura, J.; Ramsteiner, M.; Geelhaar, L.; Riechert, H. Si Doping Effects on (In,Ga)N Nanowires J. Appl. Phys. 2014, 116, 244310.

(35) Fang, Z.; Robin, E.; Rozas-Jiménez, E.; Cros, A.; Donatini, F.; Mollard, N.; Pernot, J.; Daudin, B. Si Donor Incorporation in GaN Nanowires Nano Lett. 2015, 15, 67946801.

(36) Wang, Y. J.; Xu, S. J.; Li, Q.; Zhao, D. G.; Yang, H. Band Gap Renormalization and Carrier Localization Effects in InGaN/GaN Quantum-wells Light Emitting Diodes with $\mathrm{Si}$ Doped Barriers Appl. Phys. Lett. 2006, 88, 041903.

(37) Zhao, S.; Fathololoumi, S.; Bevan, K. H.; Liu, D. P.; Kibria, M. G.; Li, Q.; Wang, G. T.; Guo, H.; Mi, Z. Tuning the Surface Charge Properties of Epitaxial InN Nanowires Nano Lett. 2012, 12, 2877-2882. 
(38) Davies, M. J.; Dawson, P.; Massabuau, F. C. P.; Oliver, R. A.; Kappers, M. J.; Humphreys, C. J. The Effects of Si-doped Prelayers on the Optical Properties of InGaN/GaN Single Quantum Well Structures Appl. Phys. Lett. 2014, 105, 092106.

(39) Chiaki, S.; Fumito, M.; Tomoaki, K.; Masahide, K.; Yasuhiro, M.; Yuji, A.; Atsushi, A. Y. Surface Morphologies and Optical Properties of Si Doped InGaN MultiQuantum-Well Grown on Vicinal Bulk GaN(0001) Substrates Jpn. J. Appl. Phys. 2013, 52, 115601.

(40) Chichibu, S.; Cohen, D. A.; Mack, M. P.; Abare, A. C.; Kozodoy, P.; Minsky, M.; Fleischer, S.; Keller, S.; Bowers, J. E.; Mishra, U. K. et al. Effects of Si-doping in the barriers of InGaN Multiquantum Well Purplish-blue Laser Diodes Appl. Phys. Lett. 1998, 73, 496-498.

(41) Guo, W.; Zhang, M.; Banerjee, A.; Bhattacharya, P. Catalyst-Free InGaN/GaN Nanowire Light Emitting Diodes Grown on (001) Silicon by Molecular Beam Epitaxy Nano Lett. 2010, 10, 3355-3359.

(42) Cho, J.; Hwang, T. Y.; Zewail, A. H. Visualization of Carrier Dynamics in p(n)type GaAs by Scanning Ultrafast Electron Microscopy Proc. Natl. Acad. Sci. 2014, 111, 20942099.

(43) Milan, S. M.; Shigefusa, C.; Siegfried, B. F.; Amber, C. A.; John, E. B.; Evelyn, L. H.; Stacia, K.; Umesh, K. M.; Steven, P. D. Optical Properties of InGaN/GaN Quantum Wells with Si Doped Barriers Jpn. J. Appl. Phys. 1998, 37, L1362-L1364.

(44) Cho, Y.-H.; Song, J. J.; Keller, S.; Minsky, M. S.; Hu, E.; Mishra, U. K.; DenBaars, S. P. Influence of Si Doping on Characteristics of InGaN/GaN Multiple Quantum Wells Appl. Phys. Lett. 1998, 73, 1128-1130.

(45) Wang, M.; Ren, F.; Zhou, J.; Cai, G.; Cai, L.; Hu, Y.; Wang, D.; Liu, Y.; Guo, L.; Shen, S. N Doping to ZnO Nanorods for Photoelectrochemical Water Splitting under Visible Light: Engineered Impurity Distribution and Terraced Band Structure Sci. Rep. 2015, 5, 12925.

(46) Tyler, W. W.; Woodbury, H. H. Scattering of Carriers from Doubly Charged Impurity Sites in Germanium Phys. Rev. 1956, 102, 647-655.

(47) Jensen, S. A.; Mics, Z.; Ivanov, I.; Varol, H. S.; Turchinovich, D.; Koppens, F. H. L.; Bonn, M.; Tielrooij, K. J. Competing Ultrafast Energy Relaxation Pathways in Photoexcited Graphene Nano Lett. 2014, 14, 5839-5845. 
(48) Wei, P.-C.; Chattopadhyay, S.; Yang, M.-D.; Tong, S.-C.; Shen, J.-L.; Lu, C.-Y.; Shih, H.-C.; Chen, L.-C.; Chen, K.-H. Room-temperature Negative Photoconductivity in Degenerate InN Thin Films with a Supergap Excitation Phys. Rev. B 2010, 81, 045306.

(49) Höpfel, R. A.; Shah, J.; Wolff, P. A.; Gossard, A. C. Electron-hole Scattering in GaAs Quantum Wells Phys. Rev. B 1988, 37, 6941-6954. 